\title{
Global Dynamics of a Higher Order Difference Equation with Quadratic Term
}

\author{
Erkan Taşdemir \\ Kırklareli University, Pınarhisar Vocational School, Kırklareli, Turkey
}

July 25, 2020

\begin{abstract}
In this paper, we investigate the dynamics of following higher order difference equation

$$
x_{n+1}=A+B \frac{x_{n}}{x_{n-m}^{2}}
$$

with $A, B$ and initial conditions are positive numbers. Especially we study the boundedness, periodicity, oscillation behaviours, global asymptotically stability and rate of convergence of related higher order difference equations.
\end{abstract}

\section{Introduction}

Difference equations and their systems have captured the attention of the researchers over the last two decades. This attention result from area of usage of difference equations. In particular they which arise in mathematical models that describe problems in ecology, probability and engineering, etc. Since we know very little about such equations, it is very important to study higher order difference equations.

In [11], Devault et al investigated the boundedness, global stability and periodic character of solutions of the difference equation

$$
x_{n+1}=p+\frac{x_{n-k}}{x_{n}}
$$

where $p$ and the initial conditions are arbitrary positive numbers.

In [13], Saleh et al investigated global asymptotic stability, periodicity and semi-cycle analysis of the unique positive equilibrium of following difference equation

$$
y_{n+1}=A+\frac{y_{n-k}}{y_{n}}
$$

where $A$ is positive and $k \in\{2,3, \cdots\}$. 
In [7], Abu-Saris et al studied the global stability of unique positive equilibrium point of following higher order difference equations

$$
y_{n+1}=A+\frac{y_{n}}{y_{n-k}}
$$

where $A$ is positive and $k \in\{2,3, \cdots\}$. Additionally, in [15], Saleh et al dealt with the global asymptotic stability of the negative equilibrium of the difference equation (1) where $A<0$ and $k \in\{1,2, \cdots\}$.

In [10], Hassan studied dynamics of following difference equation

$$
x_{n+1}=p x_{n}+\frac{q}{x_{n-1}^{2}}
$$

where $p$ and $q$ lie in $(0,1)$.

In [9], Bešo et al investigated boundedness, global attractivity and NeimarkSacker bifurcation of following difference equation

$$
x_{n+1}=\gamma+\delta \frac{x_{n}}{x_{n-1}^{2}}
$$

where $\gamma, \delta$ are positive real numbers and the initial conditions are positive real numbers.

Motivated by the above studies, we study the dynamics of following higher order difference equation

$$
x_{n+1}=A+B \frac{x_{n}}{x_{n-m}^{2}}
$$

where $A, B$ are positive real numbers and the initial conditions are positive numbers. Additionally, we investigate the boundedness, periodicity, oscillation behaviours, global asymptotically stability and rate of convergence of related higher order difference equations.

Now, we present some important theorems which used by us during this study.

Theorem 1 (See [4]) Assume that $q_{i} \in \mathbb{R}, i=1,2, \cdots$, and $k \in\{0,1, \cdots\}$. Then

$$
\sum_{i=0}^{k}\left|q_{i}\right|<1
$$

is a sufficient condition for the asymptotic stability of the difference equation

$$
x_{n+k}+q_{1} x_{n+k-1}+\ldots+q_{k} x_{n}=0, n=0,1, \cdots .
$$

Theorem 2 ([12], p. 18) Let $f:[a, b]^{k} \rightarrow[a, b]$ be a continuous function, where $k$ is a positive integer, and where $[a, b]$ is an interval of real numbers and consider the following difference equation

$$
x_{n+1}=f\left(x_{n}, \cdots, x_{n-k}\right), n=0,1, \cdots .
$$

Suppose that $f$ satisfies the following conditions: 
i. For each integer $i$ with $1 \leq i \leq k+1$, the function $f\left(z_{1}, z_{2}, \cdots, z_{k+1}\right)$ is weakly monotonic in $z_{i}$ for fixed $z_{1}, z_{2}, \cdots, z_{i-1}, z_{i+1}, \cdots, z_{k+1}$.

ii. If $(m, M)$ is a solution of the system

$$
m=f\left(m_{1}, m_{2}, \cdots, m_{k+1}\right) \text { and } M=f\left(M_{1}, M_{2}, \cdots, M_{k+1}\right),
$$

then $m=M$, where for each $i=1,2, \cdots, k+1$, we set

$$
m_{i}=\left\{\begin{array}{l}
m, \text { if } f \text { nondecreasing in } z_{i}, \\
M, \text { if } f \text { nonincreasing in } z_{i},
\end{array}\right\}
$$

and

$$
M_{i}=\left\{\begin{array}{l}
M, \text { if } f \text { nondecreasing in } z_{i}, \\
m, \text { if } f \text { nonincreasing in } z_{i} .
\end{array}\right\}
$$

Then there exists exactly one equilibrium point $\bar{x}$ of the difference equation (3), and every solution of (3) converges to $\bar{x}$.

Theorem 3 (See [8]) Let $n \in N_{n_{0}}^{+}$and $g(n, u, v)$ be a decreasing function in $u$ and $v$ for any fixed $n$. Suppose that for $n \geq n_{0}$, the inequalities

$$
y_{n+1} \leq g\left(n, y_{n}, y_{n-1}\right) \leq u_{n+1}
$$

hold. Then

$$
y_{n_{0}-1} \leq u_{n_{0}-1}, y_{n_{0}} \leq u_{n_{0}}
$$

implies that

$$
y_{n} \leq u_{n}, n \geq n_{0}
$$

Consider the scalar $k$ th-order linear difference equation

$$
x(n+k)+p_{1}(n) x(n+k-1)+\cdots+p_{k}(n) x(n)=0,
$$

where $k$ is a positive integer and $p_{i}: \mathbb{Z}^{+} \rightarrow \mathbb{C}$ for $i=1, \cdots, k$. Assume that

$$
q_{i}=\lim _{k \rightarrow \infty} p_{i}(n), i=1, \cdots, k,
$$

exist in $\mathbb{C}$. Consider the limiting equation of (4):

$$
x(n+k)+q_{1} x(n+k-1)+\cdots+q_{k} x(n)=0 .
$$

Theorem 4 (Poincaré's Theorem) Consider (4) subject to condition (5). Let $\lambda_{1}, \cdots, \lambda_{k}$ be the roots of the characteristic equation

$$
\lambda^{k}+q_{1} \lambda^{k-1}+\cdots+q_{k}=0
$$

of the limiting equation (6) and suppose that $\left|\lambda_{i}\right| \neq\left|\lambda_{j}\right|$ for $i \neq j$. If $x(n)$ is a solution of (4), then either $x(n)=0$ for all large $n$ or there exists an index $j \in\{1, \cdots, k\}$ such that

$$
\lim _{n \rightarrow \infty} \frac{x(n+1)}{x(n)}=\lambda_{j} .
$$


The following results were obtained by Perron, and one of Perron's results was improved by Pituk, see [17].

Theorem 5 Suppose that (5) holds. If $x(n)$ is a solution of (4), then either $x(n)=0$ eventually or

$$
\lim _{n \rightarrow \infty} \sup \left(\left|x_{j}(n)\right|\right)^{1 / n}=\lambda_{j} .
$$

where $\lambda_{1}, \cdots, \lambda_{k}$ are the (not necessarily distinct) roots of the characteristic equation (7).

Firstly, we take the change of the variables for Eq.(2) as follows $y_{n}=\frac{x_{n}}{A}$. From this, we obtain the following difference equation

$$
y_{n+1}=1+p \frac{y_{n}}{y_{n-m}^{2}}
$$

where $p=\frac{B}{A^{2}}$. From now on, we handle the difference equation (8). The unique positive equilibrium point of Eq.(8) is

$$
\bar{y}=\frac{1+\sqrt{1+4 p}}{2} .
$$

\section{Periodicity of Eq.(8)}

In this section, we study the periodic solutions of Eq.(8) with period two.

Theorem 6 Let $\left\{y_{n}\right\}$ be a positive solution of Eq.(8). Then Eq.(8) has no two periodic solution.

Proof. We assume that there exist two periodic solution such that

$$
\cdots, \alpha, \beta, \alpha, \beta, \cdots
$$

where $\alpha$ and $\beta$ are positive and distinct real numbers. We handle two cases for the proof of Theorem. Firstly we consider a case such that $m$ is even. We have from Eq.(8)

$$
\alpha=1+\frac{p}{\beta}, \beta=1+\frac{p}{\alpha} .
$$

Hence we obtain that

$$
\alpha^{2}-\alpha-p=0 .
$$

So we get $\alpha=\frac{1+\sqrt{1+4 p}}{2}=\bar{y}=\beta$ which is a trivial solution. Now we deal with the other case such that $m$ is odd. Now we apply Elsayed's new method for two periodic solution, see [16]. We have from Eq.(8)

$$
\alpha=1+\frac{p \beta}{\alpha^{2}}, \beta=1+\frac{p \alpha}{\beta^{2}} .
$$


Hence if we take $\alpha=\beta n$ for $n \in \mathbb{R}-\{0,1,-1\}$. Therefore we obtain that

$$
\begin{aligned}
\beta n & =1+\frac{p}{n^{2} \beta}, \\
\beta & =1+\frac{p n}{\beta} .
\end{aligned}
$$

Thus, subtracting (10) from (9) gives the following

$$
\beta(n-1)=\frac{p}{\beta}\left(\frac{1}{n^{2}}-n\right)=\frac{p}{\beta} \frac{1-n^{3}}{n^{2}} .
$$

From $n \neq 1$, we have

$$
\begin{aligned}
\beta^{2} & =\frac{-p\left(n^{2}+n+1\right)}{n^{2}}, \\
\beta & = \pm \sqrt{\frac{-p\left(n^{2}+n+1\right)}{n^{2}}} .
\end{aligned}
$$

Since $\beta$ is real number, (11) is impossible for all real $n$ and $p>0$. This is a contradiction. So the proof is completed.

\section{Boundedness of Eq.(8)}

This section, we investigate the bounded solutions of Eq.(8).

Theorem 7 Let $0<p<1$. Then every solutions of Eq.(8) is bounded and persist such that

$$
1<y_{n} \leq \frac{1-p^{n}}{1-p}+p^{n-1} C_{1}
$$

where $C_{1}=p\left(y_{0}+\frac{1}{p-1}\right)$.

Proof. Let $\left\{y_{n}\right\}$ be a positive solution of Eq.(8) and $p>0$. Then, we have from Eq. (8)

$$
\begin{aligned}
& y_{1}=1+p \frac{y_{0}}{y_{-m}^{2}}>1 \\
& y_{2}=1+p \frac{y_{1}}{y_{1-m}^{2}}>1
\end{aligned}
$$

Thus we obtain by induction $y_{n}>1$ for $n \geq 1$.

Now we consider the other side. We have from Eq.(8)

$$
y_{n+1}=1+p \frac{y_{n}}{y_{n-m}^{2}} \leq 1+p y_{n} .
$$

According to Theorem 3 , there exist a sequence $y_{n} \leq u_{n}, n=0,1, \cdots$, where $\left\{u_{n}\right\}$ satisfies

$$
u_{n+1}=1+p u_{n}, n \geq 1,
$$


such that $u_{s}=y_{s}, u_{s+1}=y_{s+1}, s \in\{-m,-m+1, \cdots\}, n \geq s$. Therefore the solution of the difference equation (13) is

$$
u_{n}=\frac{1-p^{n}}{1-p}+p^{n-1} C_{1}
$$

where $C_{1}=p\left(y_{0}+\frac{1}{p-1}\right)$. Moreover we obtain from (12) and (14),

$$
y_{n+1}-u_{n+1} \leq p\left(y_{n}-u_{n}\right)
$$

where $n>s$ and $p \in(0,1)$. So, we get $y_{n} \leq u_{n}, n>s$ as desired.

\section{Stability of Eq.(8)}

In this here, we study the stability of Eq.(8). Firstly we handle the linearized equation of Eq.(8) about its unique positive equilibrium point. Let $I$ be some interval of real numbers and let

$$
f: I^{m+1} \rightarrow I
$$

be a continuously differentiable function such that $f$ is defined by

$$
f\left(y_{n}, y_{n-1}, \cdots, y_{n-m}\right)=1+p \frac{y_{n}}{y_{n-m}^{2}} .
$$

Therefore we have

$$
\begin{gathered}
q_{0}=\frac{\partial f}{\partial y_{n}}=\frac{p}{\bar{y}^{2}} \\
q_{1}=q_{2}=\cdots=q_{m-1}=0, \\
q_{m}=\frac{\partial f}{\partial y_{n-m}}=-\frac{2 p}{\bar{y}^{2}} .
\end{gathered}
$$

Hence we obtain the linearized equation of Eq.(8) about its unique positive equilibrium point $\bar{y}$ as follow:

$$
z_{n+1}-\frac{p}{\bar{y}^{2}} z_{n}+\frac{2 p}{\bar{y}^{2}} z_{n-m}=0 .
$$

Therefore, the characteristic equation of Eq.(8) is

$$
\lambda^{m+1}-\frac{p}{\bar{y}^{2}} \lambda^{m}+\frac{2 p}{\bar{y}^{2}}=0 .
$$

Theorem 8 Let $p \in\left(0, \frac{3}{4}\right)$. Then the equilibrium point $\bar{x}$ of Eq.(8) is locally asymptotically stable. 
Proof. From (15), we have

$$
\left|q_{1}\right|+\left|q_{2}\right|+\cdots\left|q_{m}\right|=\frac{3 p}{\bar{y}^{2}} .
$$

Note that

$$
\frac{p}{\bar{y}^{2}}=\frac{2 p+1-\sqrt{4 p+1}}{2 p}
$$

Thus

$$
\begin{aligned}
\left|q_{1}\right|+\left|q_{2}\right|+\cdots\left|q_{m}\right| & =\frac{3 p}{\bar{y}^{2}}<1, \\
\frac{3(2 p+1-\sqrt{4 p+1})}{2 p} & <1, \\
\frac{4 p+3-3 \sqrt{4 p+1}}{2 p} & <0 .
\end{aligned}
$$

Hence, we get from $p>0$,

$$
(\sqrt{4 p+1}-1)(\sqrt{4 p+1}-2)<0 .
$$

So, we obtain $0<p<\frac{3}{4}$. Therefore, the proof of Theorem 8 is completed.

Theorem 9 Let $0<p<\frac{3}{4}$. Then the equilibrium point $\bar{y}$ of Eq.(8) is globally asymptotically stable.

Proof. Firstly, we consider the following function

$$
f(u, v)=f\left(y_{n}, y_{n-m}\right)=1+p \frac{y_{n}}{y_{n-m}^{2}} .
$$

The function $f(u, v)$ is nondecreasing in $u$ and nonincreasing in $v$. Let $(m, M)$ is a solution of the system

$$
m=f(m, M) \text { and } M=f(M, m) .
$$

Hence we obtain that

$$
m=1+p \frac{m}{M^{2}}, M=1+p \frac{M}{m^{2}} .
$$

Therefore we have $m=M$. According to Theorem 2, every solution of Eq.(8) converges to $\bar{x}$, as desired.

\section{Rate of Convergence of Eq.(8)}

In this section, we handle the rate of convergence of Eq.(8). 
Theorem 10 Every solution of Eq.(8) satisfies both of the following asymptotic relations

$$
\begin{aligned}
\lim _{n \rightarrow \infty}\left|\frac{y_{n+1}-\bar{y}}{y_{n}-\bar{y}}\right| & =\left|\lambda_{j}\right|, \\
\lim _{n \rightarrow \infty} \sup \left(\left|y_{n}-\bar{y}\right|\right)^{1 / n} & =\left|\lambda_{j}\right|
\end{aligned}
$$

where $j \in\{1, \cdots, k\}$ and $\lambda_{j}$ are the roots of characteristic equation (16).

Proof. We get from Eq.(8):

$$
\begin{aligned}
y_{n+1}-\bar{y} & =\left(1+p \frac{y_{n}}{y_{n-m}^{2}}\right)-\left(1+p \frac{\bar{y}}{\bar{y}^{2}}\right) \\
& =\frac{p}{y_{n-m}^{2}}\left(y_{n}-\bar{y}\right)-\frac{p\left(y+y_{n-m}\right)}{\bar{y} \cdot y_{n-m}^{2}}\left(y_{n-m}-\bar{y}\right) .
\end{aligned}
$$

Set $e_{n}=y_{n}-\bar{y}$. Therefore we have

$$
e_{n+1}+p_{n} e_{n}+q_{n} e_{n-m}=0
$$

where

$$
p_{n}=-\frac{p}{y_{n-m}^{2}}, q_{n}=\frac{p\left(y+y_{n-m}\right)}{\bar{y} \cdot y_{n-m}^{2}} .
$$

Due to the equilibrium point $\bar{y}$ of Eq.(8) is globally asymptotically stable, we get

$$
\lim _{n \rightarrow \infty} p_{n}=-\frac{p}{\bar{y}^{2}}, \lim _{n \rightarrow \infty} q_{n}=\frac{2 p}{\bar{y}^{2}} .
$$

Hence, the limiting equation of Eq.(8) is the linearized equation (15).

\section{A Numerical Simulation}

This section, we present a numerical example for verify our theoretical results.

Example 11 Consider Eq.(8) for $m=5$ and $p=0.7$. Hence we have following difference equation

$$
y_{n+1}=1+0.7 \frac{y_{n}}{y_{n-5}^{2}} .
$$

Let the initial conditions are $y_{-5}=10, y_{-4}=6, y_{-3}=8, y_{-2}=4, y_{-1}=$ 3 and $y_{0}=5$. Then unique positive equilibrium point of Eq.(17) is globally asymptotically stable. See Figure 1. 


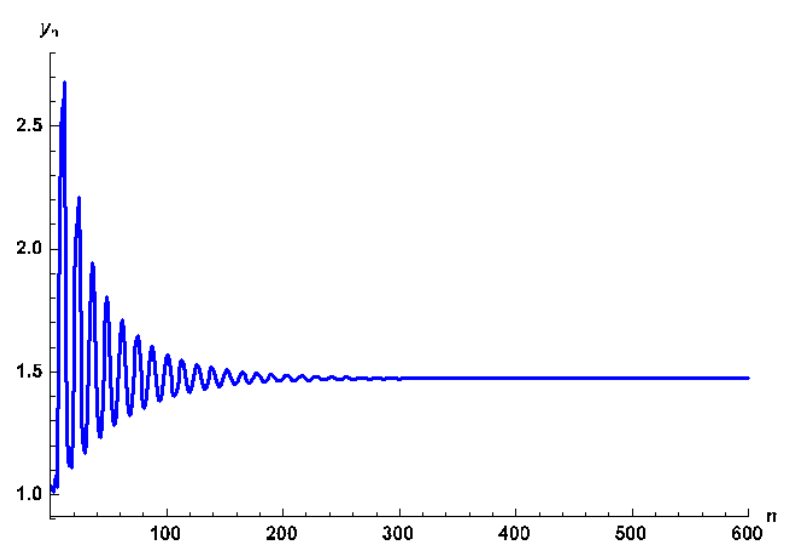

Figure 1: Plot of Eq.(17).

\section{Conclusion and Open Problems}

During this paper, we investigate the dynamics of difference equation (8). We firstly find out that Eq.(8) has not periodic solution with period two. Then we reveal the bounded solution Eq.(8). Moreover, we discover that the equilibrium point $\bar{y}$ of Eq.(8) is globally asymptotically stable. Additionally, we study the rate of convergence of Eq.(8). Finally we present an example in order to verify our theoretical results.

Open Problem 1: Investigate the dynamics of following higher order difference equation

$$
x_{n+1}=A+B \frac{x_{n}}{x_{n-m}^{r}}
$$

where the initial values are real numbers and $r \in\{3,4, \cdots\}$.

Open Problem 2: Investigate the dynamics of following higher order difference equation

$$
x_{n+1}=A+B \frac{x_{n}^{q}}{x_{n-m}^{r}}
$$

where the initial values are real numbers and $r \in\{2,3, \cdots\}, q \in\{2,3, \cdots\}$.

\section{References}

[1] S. Elaydi, An Introduction to Difference Equations, Springer-Verlag, New York, 1996.

[2] M.R.S. Kulenovic, G. Ladas, Dynamics of Second Order Rational Difference Equations with Open Problems and Conjectures, Chapman \& Hall/CRC, Boca Raton, 2002 
[3] E. Camouzis, G. Ladas, Dynamics of third order rational difference equations with open problems and conjectures, volume 5 of Advances in Discrete Mathematics and Applications, Chapman \& Hall/CRC, Boca Raton, 2008.

[4] V.L. Kocic, G. Ladas, Global Asymptotic Behavior of Nonlinear Difference Equations of Higher Order with Applications, Kluwer Academic Publishers, Dordrecht, 1993.

[5] S.A. Kuruklis, The asymptotic stability of $x n+1$ axn + bxn $k=0$, J. Math. Anal. Appl. 188 (1994) 719-731.

[6] V.G. Papanicolaou, On the asymptotic stability of a class of linear difference equations, Math. Mag. 69 (1996) 34-43.

[7] R. Abu-Saris, R. DeVault, Global stability of yn+1= A+ yn/yn- k, Appl. Math. Lett. 16 (2003) 173-178.

[8] A. Bilgin and M.R.S. Kulenovic, Global asymptotic stability for discrete single species population models, Discrete Dyn. Nat. Soc., 2017 (2017) 115 .

[9] E. Bešo, S. Kalabušić, N. Mujić, E. Pilav, Boundedness of solutions and stability of certain second-order difference equation with quadratic term. Adv. Differ. Equ. 2020(19) (2020) 1-22. https://doi.org/10.1186/s13662019-2490-9

[10] S.S. Hassan, Dynamics of the Rational Difference Equation $\quad \mathrm{xn}+1=\mathrm{pxn}+\mathrm{qx} 2 \mathrm{n}-1 . \quad$ Preprints 2020, 2020040113 (doi: 10.20944/preprints202004.0113.v1).

[11] R. DeVault, C. Kent, W. Kosmala, On the recursive sequence $\mathrm{xn}+1=\mathrm{p}+(\mathrm{xn}-\mathrm{k} / \mathrm{xn})$, J. Difference Equ. Appl., 9 (8) (2003) 721-730.

[12] E.A. Grove, G. Ladas, Periodicities in Nonlinear Difference Equations, Vol. 4, Chapman \& Hall / CRC, 2005.

[13] M. Saleh, M. Aloqeili, On the rational difference equation $y n+1=\mathrm{A}+$ yn-k yn , Appl. Math. Comput. 171(1) (2005) 862-869.

[14] M. Saleh, S. Abu-Baha, Dynamics of a higher order rational difference equation. Appl. Math. Comput. 181 (2006) 84-102.

[15] M. Saleh, M. Aloqeili, On the difference equation yn $+1=\mathrm{A}+\mathrm{yn} / \mathrm{yn}-\mathrm{k}$ with $\mathrm{A}<0$. Appl. Math. Comput. 176(1) (2006) 359-363.

[16] E.M. Elsayed, New method to obtain periodic solutions of period two and three of a rational difference equation, Nonlinear Dyn., 79 (2015) 241-250.

[17] M. Pituk, More on Poincaré's and Perron's theorems for difference equations, J. Difference Equ. Appl. 8(3) (2002) 201-216. 
[18] Y.H. Su, W.T. Li, Global attractivity of a higher order nonlinear difference equation, J. Difference Equ. Appl. 11(10) (2005) 947-958.

[19] E.M.E. Zayed, M.A. El-Moneam, On the rational recursive two sequences $\mathrm{xn}+1=\mathrm{axn}-\mathrm{k}+\mathrm{bxn}-\mathrm{k} /(\mathrm{cxn}+\mathrm{dxn}-\mathrm{k})$, Acta Math. Vietnamica, 35 (2010) 355369. 